\title{
Spontaneous Subclavian Artery Dissection Presenting as Posterior Circulation Infarction
}

\author{
Kipyoung Jeon, $\mathrm{MD}^{1}$ and Hyun-Ji Cho, MD, PhD ${ }^{2}$ \\ 'Department of Neurology, Yeouido St. Mary's Hospital, College of Medicine, The Catholic University of Korea, Seoul, ${ }^{2}$ Department of Neurology, Incheon St. \\ Mary's Hospital, College of Medicine, The Catholic University of Korea, Incheon, Korea
}

Background: Spontaneous subclavian artery dissection (SAD) is rare. Moreover, there are very few case reports which document spontaneous SAD accompanied by symptomatic neurological deficits related with ischemic stroke.

Case Report: A 71-year-old man without any reported history of trauma presented with left facial hypesthesia, left arm ataxia and gait disturbance, subsequently diagnosed as posterior circulation infarction affecting medulla and cerebellum. The computed tomography angiography revealed spontaneous left SAD with left vertebral artery occlusion, atherosclerotic plaque surrounding the origin of left subclavian artery and normal aortic vasculature. His neurological status did not deteriorate further after dual anti-platelet therapy, and intravenous hydration with volume expander were applied.

Conclusions: This is an unusual case of spontaneous SAD with lateral medullary infarction as well as cerebellar infarction. Although spontaneous SAD is a distinctly rare disease entity, it should be considered in the differential diagnosis when multiple posterior circulation infarctions are observed.

J Neurocrit Care 2017;10(2):116-121

Key words: Subclavian artery; Dissection; Cerebral infarction

\author{
Received June 23, 2017 \\ Revised September 12, 2017 \\ Accepted October 13, 2017 \\ Corresponding Author: \\ Hyun-Ji Cho, MD, PhD \\ Department of Neurology, Incheon St. \\ Mary's Hospital, College of Medicine, \\ The Catholic University of Korea, 56 \\ Dongsu-ro, Bupyeong-gu, Incheon \\ 21431, Korea \\ Tel: $+82-32-280-5010$ \\ Fax: +82-32-280-6442 \\ E-mail: fairlife1071@gmail.com
}

Copyright $\odot 2017$ The Korean Neurocritical Care Society

\section{INTRODUCTION}

Spontaneous subclavian artery dissection (SAD) has been rarely described in the relevant medical literature. ${ }^{1-3} S A D$ has been reported with aortic anomalies including coarctation of the aorta, intrathoracic subclavian artery aneurysm and/or Marfan syndrome $e^{3,4}$ or secondary to blunt trauma or iatrogenic injury during catheterization in an anatomically normal aortic arch. ${ }^{5,6}$

The main clinical manifestations of SAD were chest pain, ${ }^{3}$ back pain, ${ }^{4}$ neck pain, ${ }^{1}$ arm pulselessness, ${ }^{2}$ dizziness with nausea and vomiting ${ }^{7-9}$ and visual disturbance. ${ }^{9}$ The complications of SAD have included ischemic stroke, ${ }^{7-9} \mathrm{arm}$ ischemia ${ }^{2}$ and subclavian steal syndrome. ${ }^{10}$ Although the ischemic stroke caused by SAD was reported as posterior circulation infarction presenting with neurological deficits such as facial hypesthesia, hemianopsia or gait ataxia, ${ }^{7-9}$ it was a condition extremely rarely seen in a patient with a normal aortic arch.

We report, herein, on the case of a 71-year-old male who presented with left facial hypesthesia, left arm ataxia and gait disturbance and showed posterior circulation in-

(cc) This is an Open Access article distributed under the terms of the Creative Commons Attribution Non-Commercial License (http://creativecommons.org/licenses/by$\mathrm{nc} / 4.0$ ) which permits unrestricted non-commercial use, distribution, and reproduction in any medium, provided the original work is properly cited. 
farction affecting left medulla and cerebellum with spontaneous SAD.

\section{CASE REPORT}

A 71-year-old man with 20 pack-year smoking history visited emergency room and complained of dizziness, nausea, left facial numbness and gait disturbance of relatively acute onset. The patient denied any history of physical trauma. His blood pressure was documented at 118/88 $\mathrm{mmHg}$ in the left arm, $118 / 80 \mathrm{mmHg}$ on the right, his heart rate was 83 beats per minute and the patient was afebrile. Neurologic examination revealed left facial and body hypesthesia, dysmetria of left upper limb and gait ataxia with left falling tendency, suggesting posterior circulation infarction.

Diffusion-weighted imaging (DWI) confirmed the pres- ence of acute multi-focal infarctions on left lateral medulla oblongata, left cerebellar hemisphere and cerebellar vermis (Fig. 1A-C). Computed tomographic angiography (CTA) including the aortic arch showed that left vertebral artery (VA) was occluded at proximal portion, and SAD was suspected in the region of the left sternoclavicular joint (Fig. $1 \mathrm{D}-\mathrm{F})$. The luminal stenosis of left subclavian artery distal to the dissected segment was observed (Fig. 1G). We found no evidence of left VA dissection or left posterior cerebellar artery (PICA) dissection on CTA images. The T2*-weighted gradient echo (GRE) image revealed no hypointense signal with blooming effect in left VA and PICA (Fig. 1H).

Physical examination and CTA did not show any features suggesting connective tissue disorders like Marfan syndrome. The patient was treated conservatively with aspirin and statin, along with intravenous hydration.

Transcranial Doppler (TCD) sonography taken on the third hospital day demonstrated that the flow in the left
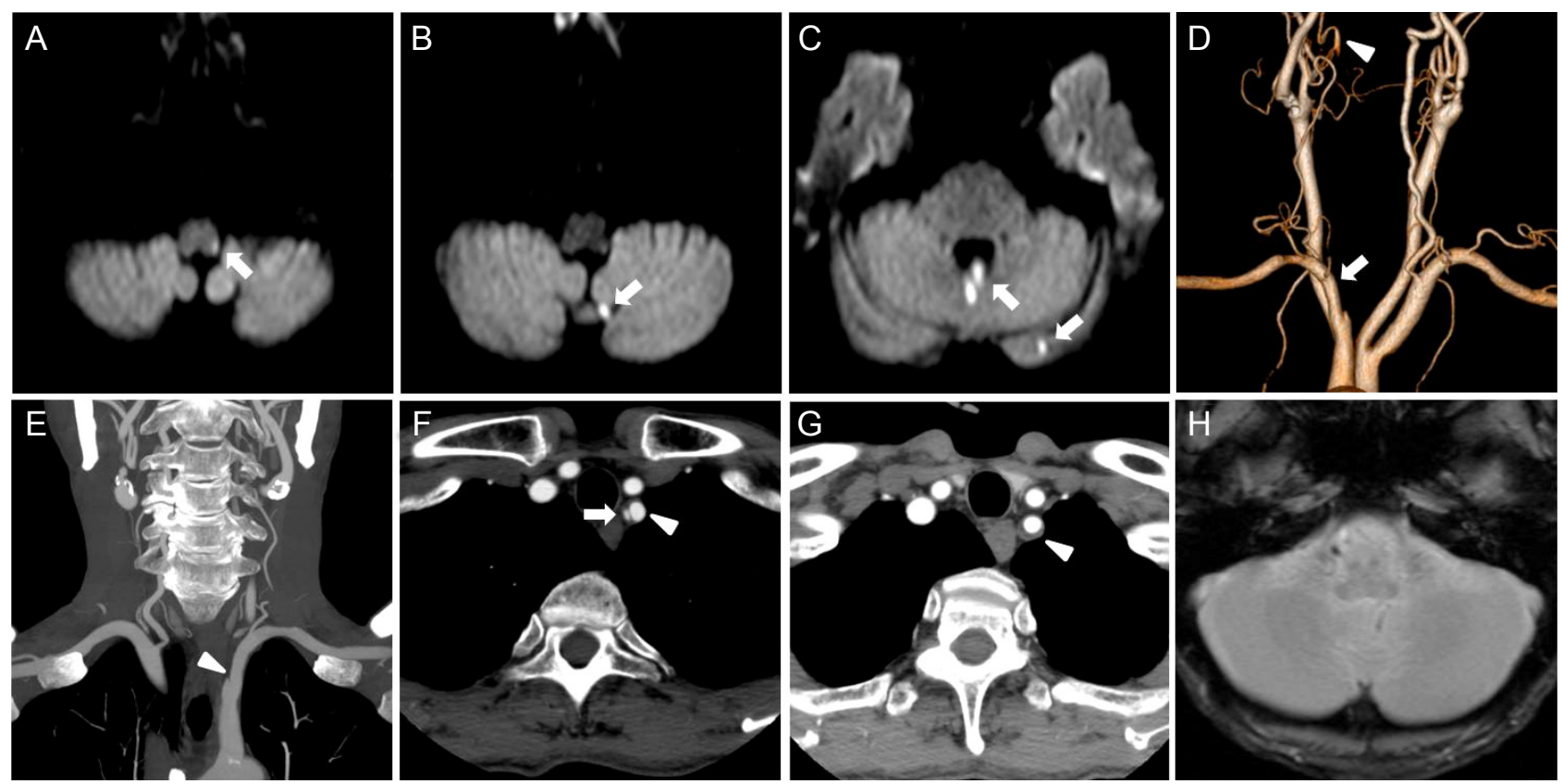

Figure 1. Brain magnetic resonance imaging and head and neck CT angiographic images taken at admission. (A-C) Initial diffusionweighted image showed acute multifocal infarctions on left lateral medulla oblongata, left cerebellar hemisphere and cerebellar vermis (white arrows). (D) Orifice of VA was not found on head and neck CT angiogram (white arrow). From distal V2 segment, contrast filling was observed (white arrowhead). (E) Short segmental dissection was suspected at left proximal subclavian artery on coronal CT image (white arrowhead). (F) Axial CT image revealed intramural hematoma (white arrow) and true lumen (white arrowhead) at left subclavian artery dissection. $(G)$ The luminal stenosis of left subclavian artery distal to the dissected segment was observed (white arrowhead). (H) T2*-weighted gradient echo image revealed no hypointense signals with blooming effect in left VA and posterior cerebellar artery. CT, computed tomography; $\mathrm{VA}$, vertebral artery. 
VA was scanty and bidirectional (Fig. 2). This result could be suggestive of the occlusion of left VA or subclavian steal syndrome. Since CTA did not show severe stenosis of left subclavian artery due to SAD, the result was possibly interpreted as high grade stenosis or occlusion of left VA. Trans- thoracic echocardiography showed normal left ventricular contractility and no evidence of intracardiac thrombus. Normal sinus rhythm with frequent premature ventricular contraction was documented by Holter monitoring. So, cardiac evaluation did not show any significant findings
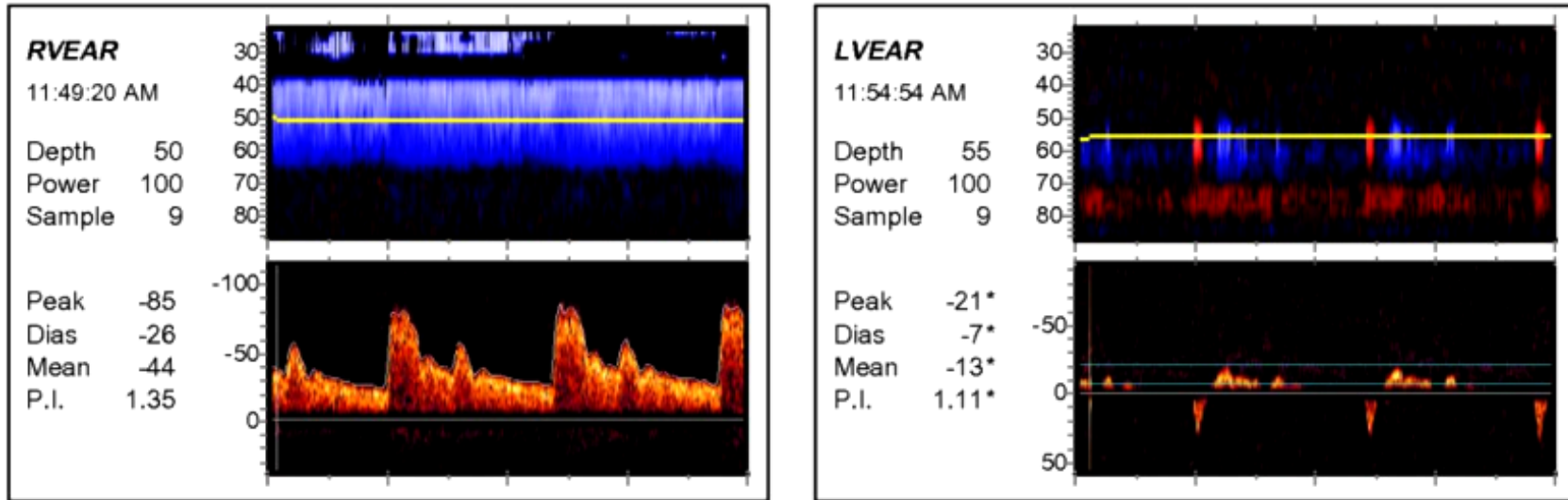

Figure 2. Transcranial Doppler findings on the third hospital day. The flow in the left proximal vertebral artery was scanty and bidirectional. Spectral analysis showed high resistance stump waveform with reversed flow.
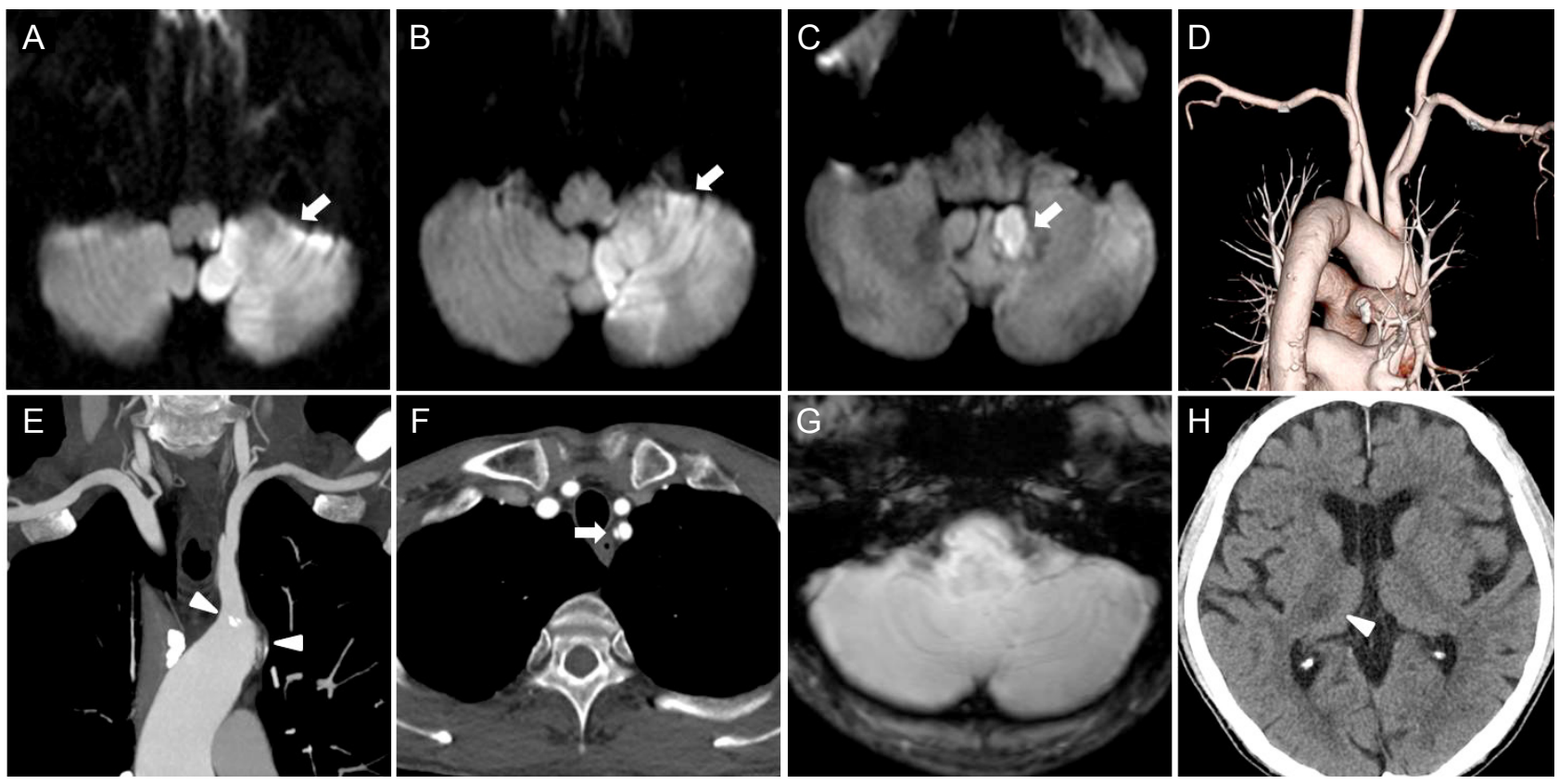

Figure 3. The Follow-up brain magnetic resonance imaging, chest CT angiographic and brain CT images taken on the fourth and fifth hospital days. (A-C) The follow-up diffusion-weighted image taken on the fourth hospital day demonstrated enlarged cerebellar hemispheric and tonsilar infarctions (white arrows). (D) No structural anomaly of aortic arch was found on CT angiogram. (E) Calcified or low density atherosclerotic plaques were found at the origin of subclavian artery on coronal CT image (white arrowheads). (F) Expansion of subintimal hematoma was not observed at left subclavian artery dissection on axial CT image (white arrow). (G) The follow-up gradient echo image showed no hypointense signal with blooming effect in left vertebral artery and posterior inferior cerebellar artery. (H) The follow-up CT scan on the fifth hospital day showed newly developed low attenuated lesion in right lateral thalamus (white arrowhead), suggesting acute infarction. CT, computed tomography. 
suggesting cardio-embolism.

On the fourth hospital day, the patient's dizziness was aggravated and he developed acute onset of tinnitus and diplopia. Neurological examination revealed dysarthria, left gaze preponderance, gaze-evoked nystagmus, right ptosis, right facial palsy and dysphagia.

The follow-up DWI showed enlarged infarcted area in left lateral medulla and cerebellum (Fig. 3A-C). The chest CTA did not discover any thrombus expansion or hematoma growth at the lesion site of left subclavian artery dissection. No structural abnormalities of aortic arch and axillary artery were observed. Atherosclerotic plaques were found around the origin of subclavian artery (Fig. 3D-F). The follow-up GRE image showed no hypo-intense signal with blooming effect in left VA and PICA, which implied that the infarct progression might not be caused by left VA or PICA dissection (Fig. 3G). The follow-up computed tomography (CT) scan on the fifth hospital day showed a newly developed, low-attenuated lesion in right lateral thalamus, suggesting acute infarction (Fig. 3H)

A volume expander and clopidogrel were added to the regimen and bed rest was recommended. The patient's neurological deficits were not aggravated further. On the eighteenth hospital day, he was discharged from the hospital and referred to an outside rehabilitation clinic for gait and balance training. Six months after discharge, all his neurological symptoms were improved except the leftsided hypesthesia and tingling sensation.

\section{DISCUSSION}

Cases of spontaneous SAD associated with ischemic stroke have been rarely reported in the past. To our knowledge, only a few cases of spontaneous SAD involved posterior circulation infarction affecting cerebellar hemisphere, cervical segments of spinal cord, thalamus and occipital lobe ${ }^{7-9}$ which was obvious because the vertebral and spinal arteries should originate from the subclavian artery.

The SAD in our patient could be characterized 'spontaneous' because he had no precipitating factors such as a re- ported history of physical trauma and/or pre-existing and underlying aortic arch anomaly. Considering that the dissected lesion at left subclavian artery was located proximal to the left sternoclavicular joint, it could have been by repetitive injury to the left shoulder and/or related musculature. Atherosclerotic plaque with calcification near the origin of left subclavian artery from the aortic arch could also represent a viable risk factor for the SAD in our patient.

The mechanism of cerebral infarction in spontaneous SAD is thought to be thrombosis or hypoperfusion or both, similar to that in carotid artery dissection. Subintimal dissection of subclavian artery could extend to VA and cause luminal stenosis or occlusion by thrombosis, or make artery-to-artery embolism to posterior circulation regions. Hypoperfusion could manifest as subclavian steal syndrome in case of severe stenosis or occlusion of subclavian artery proximal to the origin of VA. ${ }^{11}$

In this case, the patient showed left lateral medullary, left cerebellar hemisphere and vermis infarctions with spontaneous SAD. As best as we could determine based upon all available information, a lateral medullary infarction with idiopathic SAD has never been formally documented. The CTA showed left SAD with subintimal hematoma, significant stenosis of subclavian artery distal to the dissected segment and left VA occlusion. The SAD did not appear to extend into the orifice of left VA. Left VA and PICA dissections could be also excluded by CTA and GRE images. ${ }^{12}$

We considered that the pathophysiology underlying ischemic stroke in this case could be artery-to-artery embolism from left subclavian artery to left VA. We surmised that the dissecting hematoma of left subclavian artery could be an embolic source in this case, but we did not exclude the possibility that the atherosclerotic plaque of left subclavian artery might be another embolic source.

On the fourth hospital day, the infarct progression occurred without any extension of the dissected segment or thrombus expansion on the follow-up CTA images. The follow-up CT scan accomplished on the fifth hospital day showed evidence of a newly-developed, low-attenuated lesion in right lateteral thalamus supplied by right posterior cerebral artery (PCA), suggesting an acute infarction. We 
surmised that the infarct progression could be caused by distal embolization from occluded left VA to left PICA and right PCA because it was well known that distal embolization of proximal stump from persistently occluded artery could be responsible for subsequent ischemic events in distal area. ${ }^{13}$ Unfortunately, we did not perform TCD monitoring test on the vertebrobasilar arteries due to certain technical constraints in our lab, such that we were unable to directly inspect micro-embolic signals.

Our patient showed neurological deterioration during aspirin monotherapy. His neurological deterioration stopped after a volume expander and a second anti-platelet agent, clopidogrel, were added to his treatment regimen. The treatment protocol for ischemic stroke associated with SAD was not well established. In previous case reports, ischemic stroke with SAD was managed conservatively with anti-coagulation or anti-platelet therapy and showed good prognosis. ${ }^{7-9}$ It remains uncertain, whether anticoagulation therapy is superior to anti-platelet therapy for the treatment of ischemic stroke with SAD. Although it is not a direct recommendation for ischemic stroke with SAD, the 2014 guidelines for stroke prevention from American stroke association suggest that in cases of ischemic stroke or transient ischemic attack (TIA) with extracranial carotid or VA dissection, a minimum three to six months of antithrombotic treatment would be reasonable (class Ila; level B) and the efficacy of anti-platelet therapy relative to anticoagulation is unknown. ${ }^{14}$

No well-circumscribed treatment plan for endovascular treatment in patients with concomitant spontaneous SAD and posterior circulation infarction has ever been reported or documented, but some SAD cases arising from blunt trauma or iatrogenic injury during catheterization have been successfully treated with balloon angioplasty and stent insertion. ${ }^{5,6}$ Endovascular treatment of subclavian artery stenosis or occlusion associated with arm ischemia, subclavian steal syndrome, ischemic stroke or TIA showed good short and long term results. ${ }^{15}$ Therefore, endovascular treatment can be considered in patients with SAD if vascular insufficiency is severe or ischemic events recur despite other forms of intensive medical therapy.
In conclusion, this is an unusual case of spontaneous SAD with lateral medullary infarction as well as cerebellar infarction without trauma history and anomaly of aortic arch. Our patient's neurological status did not deteriorate further after dual anti-platelet therapy, and intravenous hydration with volume expander were applied. Although spontaneous SAD is a distinctly rare disease entity, it should be considered as a differential diagnosis in any case of multiple posterior circulation infarctions, unexplained neck or thoracic pain, or arm ischemic symptoms even if there was no trauma history. We suggest that the etiologic workup for posterior circulation infarction should include close scrutiny and examination of the proximal vessels emanating from the aortic arch.

\section{Conflicts of Interest}

The authors have no financial conflicts of interest.

\section{REFERENCES}

1. Marik PE, McLaughlin MT. Spontaneous subclavian artery dissection: a pain in the neck diagnosis. BMJ Case Rep 2013;2013:bcr2013201223.

2. Ananthakrishnan G, Bhat R, Zealley I. Spontaneous subclavian artery dissection causing ischemia of the arm: diagnosis and endovascular management. Cardiovasc Intervent Radiol 2009;32:326-8.

3. Henderson RA, Ward C, Campbell C. Dissecting left subclavian artery aneurysm: an unusual presentation of coarctation of the aorta. Int / Cardio/ 1993;40:69-70.

4. Stanley GA, Arko FR 3rd, Foteh MI, Jessen ME, DiMaio JM. Hybrid endovascular treatment of an anomalous right subclavian artery dissection in a patient with Marfan syndrome. Ann Thorac Surg 2012;94:639-41.

5. Spies C, Fergusson D. Treatment of an iatrogenic subclavian artery dissection. Catheter Cardiovasc Interv 2010;76:35-8.

6. Günday M, Ozülkü M, Yıldırım E, Güven A, Ciftçi O. Successful endovascular treatment of subclavian artery dissection after compression trauma. Am J Emerg Med 2013;31:457. e1-3.

7. Garewal M, Selhorst JB. Subclavian artery dissection and triple infarction of the nervous system. Arch Neurol 2005;62:1917-9. 
8. Iwamuro Y, Nakahara I, Tanaka M, Higashi T, Watanabe Y, Harada K, et al. Occlusion of the vertebral artery secondary to dissection of the subclavian artery--case report. Neurol Med Chir (Tokyo) 2005:45:97-9.

9. Fernandes AF, Lange MC, Piovesan EJ, Zamproni LN, Germiniani FM, Zétola VF. Spontaneous subclavian artery dissection in a young woman with migraine: an unusual etiology of stroke. Arq Neuropsiquiatr 2010;68:475-6.

10. Schmitter SP, Marx M, Bernstein R, Wack J, Semba CP, Dake MD. Angioplasty-induced subclavian artery dissection in a patient with internal mammary artery graft: treatment with endovascular stent and stent-graft. AJR Am / Roentgenol 1995; 165:449-51

11. Reivich M, Holling HE, Roberts B, Toole JF. Reversal of blood flow through the vertebral artery and its effect on cerebral circulation. N Engl/ Med 1961;265:878-85.

12. Park MG, Choi JH, Yang TI, Oh SJ, Baik SK, Park KP. Sponta- neous isolated posterior inferior cerebellar artery dissection: rare but underdiagnosed cause of ischemic stroke. / Stroke Cerebrovasc Dis 2014;23:1865-70

13. Pessin MS, Daneault N, Kwan ES, Eisengart MA, Caplan LR. Local embolism from vertebral artery occlusion. Stroke 1988; 19:112-5.

14. Kernan WN, Ovbiagele B, Black HR, Bravata DM, Chimowitz MI, Ezekowitz MD, et al. Guidelines for the prevention of stroke in patients with stroke and transient ischemic attack: a guideline for healthcare professionals from the American Heart Association/American Stroke Association. Stroke 2014:45:2160-236.

15. Wada T, Takayama K, Taoka T, Nakagawa H, Myouchin $K$, Miyasaka T, et al. Long-term treatment outcomes after intravascular ultrasound evaluation and stent placement for atherosclerotic subclavian artery obstructive lesions. Neuroradiol / 2014;27:213-21. 